\title{
Hssociation of HDL/TC Ratio as an Insulin Resistance Marker with Various Levels of Fasting Blood Glucose
}

\author{
Maryam Moradi BinaBaj (PhD) \\ Biochemistry of Nutrition Research Center, \\ Faculty of Medicine, Mashhad University of \\ Medical Sciences, Mashhad, Iran \\ Mohadeseh Namjoo (MSc) \\ Laboratory Sciences Research Center, Golsetan \\ University of Medical Sciences, Gorgan, Iran \\ Mojgan Nejabat (MSc) \\ School of pharmacy, Mashhad university of \\ Midical Science.Mashhad, Iran \\ Hamid Reza Joshaghani (PhD) \\ Laboratory Sciences Research Center, Golestan \\ University of Medical Sciences, Gorgan, Iran \\ Corresponding Author: Hamid Reza \\ Joshaghani \\ Email: joshaghani@goums.ac.ir
}

Tel: +98(17)32423093

Address: Department of Clinical Biochemistry, Laboratory Sciences Research Center, Golestan University of Medical Sciences, Ira

Received : 23Nov2014

Final edit: 01Dec2014

Accepted: 09Dec2014

\begin{abstract}
Background and Objective: The association of Triglyceride/High Density Lipoprotein-(holesterol (TG/HIJL-C) ratio with fasting serum insulin, which is an alternative method of insulin resistance (IR) measurement, is well-recognized. Thus, the measurement of TG/HDL-C ratio is useful to determine both IR and dyslipidemia, which itself is a characteristic of individuals with IR. Therefore, this study aimed to investigate the relationship between TG/HDL ratio as an indicator of IR, with different fasting blood glucose levels.
\end{abstract}

Methods: This case-control study was performed on 343 volunteers with no history of diabetes or use of blood glucose-lowering medications and fasting blood sugar (FBS) levels of less than $126 \mathrm{mg} / \mathrm{dl}$. After sampling, the subjects were divided into three groups based on their FBS level. First group included healthy subjects with FBS of less than $100 \mathrm{mg} / \mathrm{dl}$. Second group consisted of subjects with impaired fasting glucose (IFG) and FBS of 110-100 $\mathrm{mg} / \mathrm{dl}$ and a third group including those with impaired glucose tolerance (IGT) and FBS of $110-125 \mathrm{mg} / \mathrm{dl}$.

Results: The amount of TG/HDL-C ratio was $3.8 \pm 2.8,4.0 \pm 2.1$ and $5.4 \pm 3.8$ for the healthy group, individuals with IFG and IGT, respectively. The TG/HIDL index was significantly different among the tested groups with no significant difference between healthy subjects and subjects with IFG. Moreover, there was a statistically significant difference between the IGT and IFG groups with healthy individuals.

Conclusion: Considering the significant increase of the TG/HDL ratio in groups with impaired glucose, using this index can be helpful in evaluation of glycemic disorder.

Keywords: Insulin resistance, fasting blood glucose, impaired glucose tolerance, impaired fasting glucose, TG/HDL ratio. 


\section{INTRODUCTION}

Insulin resistance (IR) is a condition in which the body demands more insulin to sustain the glucose levels in the normal range (1). IR is associated with type II diabetes, metabolic syndrome and cardiovascular disease. Increased incidence of these disorders in Asian population is attributed to lifestyle changes and dietary patterns (2). Metabolic disorders including dyslipidemia (hypertriglyceridemia, decreased HDL-C, increased LDL) are significantly associated with IR. IR should be assessed clinically in cases of abdominal obesity, hypertension, dyslipidemia, family history of type II diabetes and in certain ethnic groups (HindiAsians and Hispanics) (3). Based on previous reports, IR has been observed even in people who are not overweight and many studies have been performed on its role as a predictor of cardiovascular disease in addition to body mass index (BMI)(4). Although many studies demonstrated the effects of dyslipidemia on the function of pancreatic beta cells in hyperglycemic individuals, its relationship in people with normal glucose tolerance should be clarified (5). Impaired fasting glucose (IFG) tolerance is one of the most common glycemic disorders and is often considered as an indicator of prediabetes (6). When impaired glucose tolerance (IGT) is detected along with elevated levels of fasting glucose or oral glucose tolerance test, a large number of beta cells have been destroyed. In this case, the early detection of IR can help prevent Type II diabetes by lifestyle modifications (7). Many studies investigated IR in people with IGT and diabetes, and limited number of studies has evaluated IR in people with normal glucose tolerance (8). There are several laboratory methods to assess IR levels and hyperinsulinemia, but the Hyperinsulinemic-euglycemic clamp technique is considered as the reference method for IR measurement. Although this method is ideal for research purposes, it is expensive, time consuming, requires special techniques and is unacceptable among patients because it involves several blood sampling phases (3). The homeostatic model assessment (HOMA) was introduced as an alternative to simplify former methods, however this method is less useful in healthy subjects compared to those with IR. Thus, researchers are seeking different ways to determine IR using various metabolic parameters (9). People in early stages of IR development have increased High Density Lipoprotein-Cholesterol (HDL-C) and Triglyceride (TG) levels (1). It is reported that the TG/HDL-C ratio is associated with fasting serum insulin (FSI) level, which is an alternative method of IR measurement. Therefore, measuring the TG/HDL-C ratio is not only useful to identify IR, it can also help the identification of dyslipidemia which itself is a characteristic of patients with IR. Due to ethnic/racial differences in TG, HDL-C and FSI levels, the relationship between TG/HDL-C with FSI differs in different ethnic groups (10). Therefore, this study was aimed to investigate the relationship between the TG/HDL ratio, as an IR indicator with different fasting blood glucose levels.

\section{MATERIAL AND METHODS}

This case-control study was done after obtaining written consent from 343 volunteers with no history of diabetes, use of blood glucose lowering drugs and FBS levels of not less than $126 \mathrm{mg} / \mathrm{dl}$.

For biochemical analysis, $5 \mathrm{ml}$ of brachial vein blood were collected after 14 hours of fasting which were immediately centrifuged at $2500 \mathrm{rpm}$. After centrifugation, serum was removed and the FBS, TG and HDL-C levels were measured by enzymatic colorimetric assay using an autoanalyzer device (Pars Azmoon kit, Pars Azmoon Co., Tehran). The subjects were then divided into three groups based on their FBS levels. The first group included healthy subjects with FBS of less than $100 \mathrm{mg} / \mathrm{dl}$, the second group included subjects with IFG and FBS of $100-110 \mathrm{mg} / \mathrm{dl}$ and the third group included people with IGT and FBS of $110-125 \mathrm{mg} / \mathrm{dl}$. The obtained data were analyzed by SPSS-18 statistical software. P-value of less than 0.05 was considered as statistically significant.

\section{RESULTS}

In this study, 140 men and 201 women, aged 16-95 were participated. Mean level of FBS, TG and HDL-C in women were 94.17, 126.49 and $37.11 \mathrm{mg} / \mathrm{dl}$ and in men were $96.74,148.25$ and $34.72 \mathrm{mg} / \mathrm{dl}$, respectively. Of the 343 participants, 258 patients (105 males and 153 females) were in the first group, 57 (22 males and 34 females) were in the second group and 28 (13 males and 14 females) patients were in the third group. 
Overall, $133(51.6 \%), 36(63.2 \%)$ and 19 patients $(67.9 \%)$ had values higher than 3 for the index, respectively. There was no statistically significant difference between the three study groups $(\mathrm{p}=0.099)$. There were significant differences between the mean of age and TG level of patients in the three groups $(\mathrm{P}=0.003)$. However, no significant difference was observed in the mean level of HDL among the three study groups. TG/HDL index was not significantly changed, but a significant difference was observed between the IGT of healthy individuals $(\mathrm{P}=0.004)$ and IGT of patients with IFG $(\mathrm{P}=0.029)$.

Table1- Comparison of the mean and P-Values between different groups (mean \pm SD)

\begin{tabular}{|c|c|c|c|c|c|c|c|c|}
\hline $\begin{array}{r}\text { P- } \\
\text { Vahue }\end{array}$ & $\begin{array}{r}\text { Index } \\
\text { TG/HDL } \\
\end{array}$ & $\begin{array}{l}\text { HDL-C } \\
\text { (mg/dl) }\end{array}$ & TG (mg/dl) & FBS (mg/dl) & Age & Gender & Groups & \\
\hline \multirow{7}{*}{ 0/004 } & \multirow{5}{*}{$3 / 8 \pm 2 / 8$} & $37 / 4 \pm 10 / 2$ & $121 / 4 \pm 79 / 4$ & $88 / 8 \pm 6 / 0$ & $42 / 6 \pm 17 / 9$ & Female & Healthy & 1 \\
\hline & & $35 / 6 \pm 17 / 3$ & $137 / 8 \pm 77 / 1$ & $90 / 1 \pm 7 / 2$ & $48 / 4 \pm 16 / 5$ & Male & & \\
\hline & & $36 / 7 \pm 13 / 5$ & $126 / 0 \pm 78 / 0$ & $89 / 8 \pm 6 / 4$ & $40 / 6 \pm 15 / 6$ & Total & \multirow{3}{*}{ IFG } & \multirow{3}{*}{2} \\
\hline & & $37 / 5 \pm 18 / 8$ & $12 / 8 \pm 41 / 8$ & $104 / 5 \pm 2 / 6$ & $51 / 2 \pm 12 / 9$ & Female & & \\
\hline & & $34 / 5 \pm 6 / 4$ & $141 / 5 \pm 77 / 6$ & $104 / 6 \pm 2 / 4$ & $52 / 7 \pm 13 / 5$ & Male & & \\
\hline & $4 / 0 \pm 2 / 1$ & $36 / 1 \pm 15 / 1$ & $142 \pm 82$ & $104 / 5 \pm 2 / 7$ & $49 / 7 \pm 15 / 1$ & Total & & \\
\hline & & $35 / 1 \pm 7 / 7$ & $167 / 1 \pm 95 / 8$ & $118 / 6 \pm 4 / 1$ & $57 / 7 \pm 10 / 6$ & Female & IGT & 3 \\
\hline \multirow{3}{*}{$0 / 029$} & \multirow{3}{*}{$5 / 4 \pm 3 / 8$} & $31 / 1 \pm 5 / 5$ & $160 / 0 \pm 97 / 4$ & $114 / 8 \pm 3 / 0$ & $55 / 4 \pm 11 / 8$ & Male & & \\
\hline & & $35 / 3 \pm 12 / 9$ & $164 \pm 100$ & $117 / 1 \pm 4 / 0$ & $57 / 1 \pm 13 / 4$ & Total & & \\
\hline & & 0/017 & $0 / 003$ & & $>0 / 001$ & & 0/099 & $\begin{array}{r}\text { P- } \\
\text { Vahue }\end{array}$ \\
\hline
\end{tabular}

\section{DISCUSSION}

This study investigated the efficacy of TG/HDL-C ratio in assessment of IR in patients with various ranges of glucose tolerance. In Gacio study, the HOMA-IR index was used to determine the amount of IR and all lipid parameters, except for HDL-C were positively associated with HOMA-IR. Compared to other lipid parameters, the relationship between HOMA-IR and TG/HDL-C was higher in the four tested ethnic groups. For each unit increase in TG/HDL-CODDS, IR increased up to 4-fold in Native Canadians, 3.4-fold in the Chinese, 1.9-fold in Europeans and 1.8-fold in South Asians. In the mentioned study, the TG/HDL$\mathrm{C}$ ratio was considered as a good marker for IR measurement in all groups except South Asians, which is in agreement with the present study (11). Sommer et al. investigated individuals with $\mathrm{BMI}=25$ and determined the IR level using $€$ index of insulin sensitivity ( $\mathrm{S} 1$ ). Individuals were divided into tertiles of 0.2 to $2.2,2.3$ to4.5, 4.3 to 12.8 and the results showed that those whose IR were clamped in the 0.2 to $2.2 \mathrm{mU} / \mathrm{L}$ range had increased FSI, but the amount of TG and
TG/HDL-C ratio did not increase. Of these, 40 patients had metabolic syndrome with only 12 in the IR tertile. Sensitivity, specificity and positive predictive value of metabolic syndrome for the diagnosis of IR in their subjects were $30 \%, 96 \%$ and $86 \%$, respectively. Also, 17 people had the lipid criteria, 7 of which were in the IR tertile. The sensitivity of this method for the determination of IR was only $17 \%$ in the mentioned study and their results showed that the TG/HDL-C ratio is not reliable for IR measurement among African-Americans. However, the present study and the aforementioned study are different in the aspect of TG increase and plasma TG/HDL-C ratio (12). Mandiya et al. studied apparently healthy individuals with recent diagnosis of IGT, IFG or both. Overall, 241 (32.2\%) of the patients had IR (HOMA-IR index of $4.4 \pm$ 1.6) in this study. The optimal amount of TyG index was determined by receiver operating characteristic (ROC) curve. The amount of TyG index gradually increased from healthy subjects to people with IGT and 
IFG or both of them, with or without IR. HOMA-IR and TyG indices showed similar trends and the amount of Pearson correlation between HOMA-IR index and TyG was 0.322 and 0.172 between HOMA-IR index and TG/HDL-C ratio. This was consistent with the findings of the present study in terms of increasing trend of the index (13). The HOMA-IR model was used to measure IR in ZiYang study on 105 women with polycystic ovary syndrome (PCOS) and 109 healthy controls. The diagnostic criteria of PCOS for individuals with IR was reported as HOMAIR of more than 2.77. Further studies showed that the TG/HDL-C, TC/HDL-C and LDL$\mathrm{C} / \mathrm{HDL}-\mathrm{C}$ are suitable diagnostic markers for people with PCOS and IR. The area under the ROC curve in TC/HDL-C was the highest with $93.2 \%$ sensitivity and $83.8 \%$ specificity. After measuring the levels of TG, glucose, fasting insulin and HDL-C, the TG/HDL-C ratio was found to be significantly correlated with HOMA-IR ( $\mathrm{P}<0.05)$, which is in agreement with our findings in terms of increased TG and plasma TG/HDL-C ratio (14). In the study of Knight et al. on 801 nondiabetic overweight African black women and 143 non-diabetic white women, after measuring the TG/HDL-C ratio and IR by the HOMA-IR method, no relationship was observed between this ratio and IR prediction. The area under the ROC curve was calculated to evaluate the predicting ability of TG/HDL$\mathrm{C}$ ratio for IR and reported as $0.76 \pm 0.06$ and $0.63 \pm 0.03$ in white and black women, respectively. In the present study, a correlation was found between the TG/HDL$\mathrm{C}$ and IR which is inconsistent with the study of Knight et al. (15). In Kim et al. study, the HOMA-IR index was found as 2.59. Similar to the present study, IR of both groups of men and women were measured by HOMA-IR which was positively correlated with TG and TG/HDL-C ratio and negatively correlated with HDL-C. Both of these correlations were statistically significant and are in agreement with our findings (16). In Tangvarasittichai et al. study on type II diabetes patients, the TG/HDL-C ratio was significantly correlated with the HOMA-IR index which is consistent with the present study findings (17). In Abbasi et al. study in 2011 on non-diabetic individuals, the area under the curve of insulin was correlated with the TG/HDL-C ratio and TyG index, which is consistent with the findings of the present study (18). In the study of Liu et al. cut-off points of 3.5, 2, 2.23 and 3 were used for the TG/HDL-C ratio in different ethnic groups and the area under the curve of this ratio for prediction of hyperinsulinemia was reported as 0.77 and 0.74 for the tested ethnic groups. The results of this study showed a significant relationship between this ratio and fasting insulin level among three ethnic groups in America. This is consistent with our findings in terms of the TG/HDL-C ratio, but no ethnical investigation was done in our study (19). In Stein et al. study on non-diabetics, a significant correlation was observed between insulin sensitivity and TG, HDL-C and TG/HDL-C ratio which are in agreement with the present study. Moreover, individuals with moderate TG levels had IR level equivalent or higher than individuals with high TG levels (20). In McLaughlin et al. study on obese non-diabetics, the optimal cut-off point for TG and TG/HDL-C ratio was 1.47 and 1.8 , respectively. The results of this study showed the useful application of the TG or TG/HDL-C ratio cut-offs in determination of IR in obese people. These results are in agreement with the present study in terms of TG and TG/HDL-C ratio measurement and their significant positive association with IR detection. However, the parameter of weight was not evaluated in our study (21). In study of Chiang et al., for each unit increase in the TG/HDL-C ratio, IR increased by $31 \%$ when evaluated by the HOMA method, which is in agreement with the findings of present study (22). In Hadaegh study in Iran, the prevalence of metabolic syndrome in individuals with TG/HDL-C ratio of $\leq 6.9$ (top quartile) and TG/HDL-C ratio of $>2.8$ (bottom quartile) was $63.6 \%$ and $3 \%$, respectively. This is consistent with our findings in terms of the TG/HDL-C ratio and its relationship with metabolic syndrome (23).

\section{CONCLUSION}

Considering the significant increase of the TG/HDL ratio in patients with impaired glucose, using this index in evaluation of glycemic disorders can be helpful. Given the dependence of this index to nutritional and genetic status, it is recommended to 
determine its cut-off by comparison with the reference methods in Iran.

\section{ACKNOWLEDGMENT}

The authors would like to thank all the participants and staff of Kavosh laboratory

\section{REFERENCES}

1. Kang HT, Yoon JH, Kim JY, Ahn SK, Linton JA, Koh SB, et al. The association between the ratio of triglyceride to $H D L-C$ and insulin resistance according to waist circumference in a rural Korean population. Nutrition, Metabolism \& Cardiovascular Diseases. 2012; 22(12): 1054-1060. doi: 10.1016/j.numecd.2011.01.013.

2. Misra A, Khurana L, Isharwal S, Bhardwaj S. South Asian diets and insulin resistance. British Journal of Nutrition. 2009; 101: 465-473.

3. Misra A, Vikram N.K. Insulin resistance syndrome (metabolic syndrome)and Asian Indians. Current Science. 2002; 83( 12): 1483-1496.

4. Kawamoto R, Tabara Y, Kohara K, Miki T, Kusunoki T, Takayama S, et al. Low-density lipoprotein cholesterol to high-density lipoprotein cholesterol ratio is the best surrogate marker for insulin resistance in non-obese Japanese adults. http://www.lipidworld.com/content/9/1/138.

5. Zheng T, Gao Y, Tian H. Relationship between blood lipid profiles and pancreatic islet $\beta$ cell function in Chinese men and women with normal glucose tolerance: a cross-sectional study. http://www.biomedcentral.com/1471-2458/12/634.

6. Erkan Coban, Ramazan Sarı. The Levels of Serum High Sensitivity C-reactiveProtein in Subjects with Impaired Fasting Glucose.Turkish Journal of Endocrinology and Metabolism, 2003; 4:165-168.

7. Dorner SJK, Deuster PA, Zeno SA, Remaley AT, Poth M. Should triglycerides and the triglycerides to high-density lipoproteincholesterol ratio be used as surrogates for insulin resistance? Metabolism Clinical and Experimental 2010; 59(2): 299-304. doi: 10.1016/j.metabol.2009.07.027.

8. Arvind Kumar, PoornimaTewari, Sibasis S. Sahoo, Arvind Kumar Srivastava. Prevalence of Insulin Resistance in First Degree Relatives of Type-2 Diabetes Mellitus Patients: A Prospective Study in North Indian Population. Indian Journal of Clinical Biochemistry. 2005; 20(2): 10-17. doi: 10.1007/BF02867394.

9. Puente BA, Disse E, Faraj M, Lavoie ME, Laville M, Rabasa-LhoretR, et al. Evaluation of insulin sensitivity with a new lipid-based index in non-diabetic postmenopausal overweight and obese womenbefore and after a weight loss intervention. European Journal of Endocrinology 2009;161(1): 51-56. doi: 10.1530/EJE-09-0091.

10. Chiang JK, Lai NS, Chang JK, Koo M. Predicting insulin resistance using the triglyceride-to-high-density lipoprotein cholesterol ratio in Taiwanese adults. This article is available from: http://www.cardiab.com/content/10/1/93. for their cooperation and assistance in this study.

\section{CONFLICT OF INTEREST}

Therer are no conflicts of interest.

11. Gasevic D, Frohlich J, Mancini GB, Lear SA. The association between triglyceride to high-densitylipoprotein cholesterol ratio and insulin resistance in a multiethnic primary prevention cohort.Metabolism.2012 ;61(4): 583-589. doi: 10.1016/j.metabol.2011.09.009.

12. Sumner AE, Harman JL, Buxbaum SG, Miller BV 3rd, Tambay AV, Wyatt SB, Taylor HA, Rotimi CN, Sarpong DF. The triglyceride/high-density lipoprotein cholesterol ratio fails to predict insulin resistance in African-American women: an analysis of Jackson Heart Study. MetabSyndrRelatDisord. 2010; 8: 511-514.

13. Luis E. Simental-Menda, Martha Rodrguez-Morn, Fernando Guerrero-Romero.The Product of Fasting Glucose and TriglyceridesAs Surrogate for Identifying Insulin Resistancein Apparently Healthy Subjects. Metabolic Syndrome And Related Disorders. 2008; 6: 299-304.

14. Shou-Kui Xiang, FeiHua, Ying Tang, Xiao-Hong Jiang, Qi Zhuang, Feng-Juan Qian. Relationship between Serum Lipoprotein Ratios and Insulin Resistance in Polycystic Ovary Syndrome.International Journal of Endocrinology 2012; 1: 1-4. doi.org/10.1155/2012/173281.

15. Knight MG, Goedecke JH, Ricks M, Evans J, Levitt NS, Tulloch-Reid MK, et al. The TG/HDL-C ratio does not predict insulin resistance in overweight women of African descent: a study of South African, African American and West African women. Ethn Dis. 2011; 21: 490-494.

16. Jong-Seok Kim, Hee-Taik Kang, Jae-Yong Shim, Hye-Ree Lee. The association between the triglyceride to high-densitylipoprotein cholesterol ratio with insulin resistance (HOMA-IR)in the general Korean population: Based on the NationalHealth and Nutrition Examination Survey in 2007-2009. Diabetes Researchand Clinical Practice. 2012; 97(1): 132-138. doi: 10.1016/j.diabres.2012.04.022.

17. Tangvarasittichai S, Poonsub P, Tangvarasittichai O. Association of serum lipoprotein ratios with insulin resistance in type 2 diabetes mellitus. Indian J Med Res. 2010; 131: 641-648.

18. Abbasi F, Reaven GM.Comparison of two methods using plasma triglyceride concentration as a surrogate estimate of insulin action in nondiabetic subjects: triglycerides $\times$ glucose versus triglyceride/high-density lipoprotein cholesterol. Metabolism. 2011; 60(12): 1673-1676. doi: 10.1016/j.metabol.2011.04.006.

19. Li C, Ford ES, Meng YX, Mokdad AH, Reaven GM. Does the association of the triglyceride to highdensity lipoprotein cholesterol ratio with fasting serum insulin differ by racelethnicity? CardiovascDiabetol. 2008; 7: 4. doi: 10.1186/1475-2840-7-4. 
20. Stein E, Kushner H, Gidding S, Falkner B.Plasma lipid concentrations in nondiabetic African American adults: associations with insulin resistance and the metabolic syndrome.Metabolism. 2007; 56: 954-960.

21. McLaughlin T, Abbasi F, Cheal K, Chu J, Lamendola C, Reaven G. Use of metabolic markers to identify overweight individuals who are insulin resistant. Ann Intern Med. 2003; 139(10): 802-9.

22. Chiang JK, Lai NS, Chang JK, Koo M. Predicting insulin resistance using the triglyceride-to-high-density lipoprotein cholesterol ratio in Taiwanese adults.CardiovascDiabetol. 2011; 10: 93. DOI: 10.1186/1475-2840-10-93.

23. Hadaegh F, Khalili D, Ghasemi A, Tohidi M, Sheikholeslami F, Azizi F. Triglyceride/HDLcholesterol ratio is an independent predictor for coronary heart disease in a population of Iranian men. NutrMetabCardiovasc Dis. 2009; 19: 401-408. doi: 10.1016/j.numecd.2008.09.003. 